Zeitschrift für Augenheilkunde 1909;22:I-VI

\title{
Contents, Vol. 22, 1909
}

\section{Inhalts -Verzeichnis}

Original-Arbeiten.

Adam, C, Studien über L·okalisation und sogenannte ,,para-

doxe Doppelbilder" bei Schielenden . 483

Ammann, E., Hornhautnarben und Sehstörungen .... 427

Asher, W., Keratitis gonorrhoica metastatica .... 435

Asmus, E., Doppelseitige Keratitis parenchymatosa auf luetischer Basis nach einseitigem Trauma 335

- --, Totale traumatische Ptosis mit Ektropium, geheiltdurch Kuhntsche Ektropium - Operation und Pagen-stechersche Suturen 222

Bach, L.i Der Sphinkterkern und die Übertragungsbahn des

Lichtreflex日s der Pupille im Vierhügel ....... 1.10

Blaauw, Eduard, Conjunctivitis vernalis $\quad 317$

Cramer, E., Beitrag zu den Erfahrungen über Augapfel-

infektionen nach Operationen (Erysipel) 211

Dy,toit, Alfr., Ein Pall von subkutaner Zerreissung des Tarsus

palpebrae sup 224

Eisner, Hans Heinrich, Klinische und experimentelle Unter-suchungen über die Ursachen von Intoxikationserschei-nungen nacli Atropinanwendung bei augenkrankenKindern 387, 507

Erdmann, P., Glaukomatöse Drucksteigerung bei Keratitis

disciformis und herpesartigen Bornhauterkiankungen . 30

Imai, S., Zur Kasuistik der ringförniigen Trübung an der

Linsenvorderfläche (Vossius) 126

Judin, K. A., Ueber die Anwendung des Thiosinamin bei

einigen Erkrankungen des Sehnerven und der Netzhaut 204

Köllner, H., Beiträge zur Pathologie des Farbensinnes. Dieerworbene Rotgrünblindheit als dichromatisches Farben-system 193, 336

$-\mathrm{IV}-$

Kramer, Richard, Das Tuberkulom der Conjunctiva bulbi nnri

seine diagnostischen Schwierigkeiten (Nachtrag) ... 54

Krusius, Franz F., Zur Dauerdrainage der abführenden

Tränenwege 230

Kuffler, Otto, Klinisch-bakteriologische Studie über Binde-haut- und Tränensackerkrankungen nebst einigen Fallenvon Panophfchalmie 405

Kuschel, J., Der akkomraodative Stauungsdriick stromaufwärts vom Gloguetsciien Kanale als örtliche Ursache der Refrak-tionserhöhung des Auges und der Kurzsichtigkeit . . 97

- -, Das Verhalten des Akkommodations-Apparates, bei

der Achsenverlängerung des Auges im besonderen . . . 283 
Langenhan, Glioma retinae des vorderen Bulbusabschnittes mit Perforation der Linsenkapsel und Abschnürung von Linsenschollen durch Tumorzellen. (Hierzu Taf. II) . . 135

Levinsohn, Georg, ZumAufsatze Backs; „Der Sphinkterkernund die Übertragungsbahn des Lichtreflexes der Pupilleim Vierhügel” 352

Mauersberg, P., Über willkürlichen Nystagmus 117

Péchin, A. und G. Ducroquet, Ueber die Bedeutung der Schrif tvom ophthalmologischen und orthopädischen Stand-punkfce 42

Pfalz, G., Über Behandlung und İSГachbehandlung von Ver-

ätzungen und Verbrennungen an den Augen 492

Pichler, Alexius, Ein Versuch, eine Form des Eigenlichtes der

Netzhaut zu erklären 304

Prediger, Hans, Über Trockenoperationen in der Augen-

heilkunde 52

Purtscher, Adolf, Netzhautriss in der Gegend der Macula bei

Abhebung der Netzhaut 215

Reis, Wilh., Notiz über den anatomischen Befund bei Ectro-

pium uveae congenitum 499

Scheuermann, Wilh., Über einen Pall von Solitärtuberkel der Netzhaut, Aderhaut und des

Sehnervenkopfes, geheilt mit Neu-Tuberkulin-Bazillen-Emulsion. (Hierzu Tafel I) 37

Stargardt, K., Über die Wirkung der Einträufelung von

Tuberkulin in den Bindehautsack 1

Werner, Ernst, Beiträge zur Frage des TVachomerregers. (Hierzu Tafel III) 321

Winselmann, G., Beitrag zur operativen Behandlung des

Astigmatismus $\quad 426$

$-\mathrm{V}-$

Beriehte über die deutsehe ophthalmologische Literatur.

Untersuchungsmethoden (I, und TT. Sem. 1908.) Von

Prof. Dr. F. Dimmer in Graz 441

Dioptrik und Anomalien der Refraktion und Akkommodation. (II. Sem. 1908.) Von Prof. Dr.

Maximilian Salzmann

in Wlen 73

I. Sera. 1909525

Spezielle Pathologie und Tlierapie. (I. und II. Sem. 1908).

$\lambda \Gamma$ on Dr. Konrad Schrader in Gera 446, 533

Anafcomie des Auges. (II. Sem. 1908.) Von Prof. Dr. Sohotta

in Würzburg 140

I. Sem. 1909436

Beriehte über die ausländisehe ophthalmologische Literatur.

Bericht über die belgische ophthalmologische Literatur (1908).

Von Dr. H. Terlinch und Dr. F. Marzorati in Brüssel 232 Bericht über die englische

ophthalmologische Literatur (1908).

Erstattet von Dr. R. Gruber in London 148

Bericht über die englische ophthalmologische Literatur.

(I. Sem. 1909.) Von Dr. Markus in London 354

Bericht über die holländische ophthalmologische Literatur

des Jahres 1908. (II. Sem.) Von Dr. G. J. Schoute in 
Amsterdam und Prof. Dr. W. Koster-Gzn in Leiden . . 156

des Jahres 1909. (I. Sem.) 234

Bericht über die russische ophthalmologische Literatur.

(I. u. II. Sem. 1908.) Von Priv.-Doz. Dr. Th. Werncke in

Odessa. (Schluss) 55

Bericht über die skandinavische ophthalmologische Literatur

(II. Sem. 1907, I. und II. Sem. 1908.) Von Priv.-Doz.

Dr. Ernst Forsmark in Stockholm 161

Gesellschaftsberiehte.

Bericht über die Verhandlungen der ophthalmologischen Sektion des XVI. internationalen medizinischen Kon-gresses in Budapest vom 30. August bis 4. September 1909. erstattet von Priv.-Doz. Dr. Scholtz in Budapest . . 242

Bericht über die ophthalmologische Sektion der 81. Versamm-lvng deufcscher Naturforseher und Ärzte in Salzburgvom 19. bis 25. September 1909, erstattet von Dr. Toldtin Salzburg 363

$-\mathrm{VI}-$

Berliner ophthalmologische Gesellschaft. Sitzung vom 17. Juni

1909 179

-- Sitzung vom 15. Juli 1909

266

Moskauer augenärztliche Gesellschaft. Sifczung vom 28. April

(11. Mai) 1909 .

81

$<--$ Sitzung vom 9. November 1909

550

St. Peterburger ophthalmologische Gesellschaft. Sitzung am

23. April und 14. Mai 1909

371

Ophthalmologische Gesellschaft in Wien. Sitzung vom

12. Mai $1909 \quad 78$

i $\quad$ Sitzung vom 16. Juni $1909 \quad 268$

Sitzung vom 27. Oktober $1909 \quad$. 543

ünfall- und Versicherungskunde. . 82, 181, 273, 372, 474.552

Therapeutische Umschau $\quad 85,182,275,375,475, \quad 553$

Diagnostische Notizen ..,_.... 87, 185, 278, 377, 478, 556Fortschritte der RöntgenundRadiumstrahlen-Forschimg 90,380,560

Aus den Grenzgebieten 90, 281

LiteraturveГzeichnis .94, 187, 381, 479, 561

Prof. Th. v. Ewetzky† 91

Aufruf für Carlo Reymond 192

Tagesnotizen $\quad 192$

Personalien 282, 386, 482, 564

S. Sachregister $\quad 565$

Namenregister $\quad 574$ 